\title{
High-efficiency CRISPR/Cas9 multiplex gene editing using the glycine tRNA-processing system-based strategy in maize
}

\author{
Weiwei Qi ${ }^{1,3}$, Tong Zhu' ${ }^{1}$, Zhongrui Tian ${ }^{1}$, Chaobin $\mathrm{Li}^{1}$, Wei Zhang ${ }^{1}$ and Rentao Song ${ }^{1,2,3^{*}}$
}

\begin{abstract}
Background: CRISPR/Cas9 genome editing strategy has been applied to a variety of species and the tRNA-processing system has been used to compact multiple gRNAs into one synthetic gene for manipulating multiple genes in rice.

Results: We optimized and introduced the multiplex gene editing strategy based on the tRNA-processing system into maize. Maize glycine-tRNA was selected to design multiple tRNA-gRNA units for the simultaneous production of numerous gRNAs under the control of one maize U6 promoter. We designed three gRNAs for simplex editing and three multiple tRNA-gRNA units for multiplex editing. The results indicate that this system not only increased the number of targeted sites but also enhanced mutagenesis efficiency in maize. Additionally, we propose an advanced sequence selection of gRNA spacers for relatively more efficient and accurate chromosomal fragment deletion, which is important for complete abolishment of gene function especially long non-coding RNAs (IncRNAs). Our results also indicated that up to four tRNA-gRNA units in one expression cassette design can still work in maize.
\end{abstract}

Conclusions: The examples reported here demonstrate the utility of the tRNA-processing system-based strategy as an efficient multiplex genome editing tool to enhance maize genetic research and breeding.

Keywords: CRISPR/Cas9, Maize, Multiplex gene editing, tRNA-processing

\section{Background}

Mutants are critical in genetic research for the study of gene function, and gene editing technologies can efficiently create mutations in targeted genes. The clustered regularly interspersed short palindromic repeat (CRISPR)/ CRISPR-associated protein (Cas) system has evolved from studies of the defense systems of bacteria to a newly established gene editing tool [1]. The CRISPR/Cas9 system is derived from Streptococcus pyogenes and possesses a protospacer adjacent motif (PAM) recognition sequence [2, 3]. The Cas9 gene and a 20-bp guide RNA (gRNA) that is complementary to the DNA site being targeted for mutation need to be transformed into the target organism to create a gene disruption. The CRISPR/Cas9 system has been demonstrated for efficient gene disruption in

\footnotetext{
* Correspondence: rentaosong@staff.shu.edu.cn

'Shanghai Key Laboratory of Bio-Energy Crops, School of Life Sciences,

Shanghai University, 333 Nanchen Road, Shanghai 200444, China

${ }^{2}$ National Maize Improvement Center of China, China Agricultural University,

Beijing 100193, China

Full list of author information is available at the end of the article
}

multiple organisms, including bacteria [1], yeast [4], zebrafish [5], fruit flies [6], human cells [7] and plants [8]. In plants, the CRISPR/Cas9 system has been effectively applied in many species, including Arabidopsis thaliana, Citrus sinensis, Nicotiana tabacum, Oryza sativa, Solanum lycopersicum, Sorghum bicolor, Triticum aestivum [9], Zea mays [10-13], and Glycine $\max$ [14]. For highly efficient gene modification, the CRISPR/Cas9 vector construction strategy should always be optimized for the usage in specific organism.

Gene editing tools with the capability to manipulate multiple targets are of great value. The CRISPR/Cas9 system is a promising tool for this purpose. Multiplex gene editing can be achieved by expressing Cas9 along with multiple gRNAs, each targeting different sites. Conventional delivery methods involve creating gene constructs containing multiple gRNA expressing cassettes for multiplex gene editing in one plasmid or using multiple plasmids [15-20]. Due to the limitations of the delivery method and plasmid capacity, compacting multiple gRNAs into one synthetic gene would be an 
advanced strategy. Xie et al. [21] demonstrated that multiple gRNAs could be efficiently produced from a single synthetic gene using a tRNA-gRNA architecture that allows for the precise excision of transcripts in vivo by the endogenous RNases, RNase P and Z, in rice. This strategy could be broadly used to generate multiplex gene editing in both monocot and dicot plants after specific optimization, because the tRNA-processing system exists in virtually all organisms.

Although the CRISPR/Cas9 system showed high efficiency for genome modification, it did not always create strong mutation with complete abolishment of gene function especially for long non-coding RNAs (lncRNAs). Chromosomal fragment deletion between target sites can be archived with multiplex gene editing strategy and constitutively improves the mutation degree [21]. As the process of gene modification by CRIPSR/Cas9 system depends on the gRNA to search and target to the specific site and the target sites meeting the requirements for targeting cannot always be targeted at the same time [5], the selection of gRNA spacers is essential for high efficient chromosomal fragment deletion.

Maize (Zea mays) is one of the most important cereal crops in the world. Here, we report our specific vector construction, sequence design and editing results of using the multiplex gene editing strategy based on the tRNA-processing system in maize. The design of the vectors was optimized for the usage in maize. We found the tRNA-processing system-based method improves the efficiency of CRISPR/Cas9 editing in maize and proposed an advanced gRNA selection strategy for chromosomal fragment deletion purpose.

\section{Methods}

\section{Plant materials}

The Hill maize parental lines PA and PB were initially obtained from the Maize Genetics Cooperation Stock Center and maintained in the lab. The transgenic lines were generated in the lab. Maize plants were cultivated in the experimental field, green house or growth chambers at the campus of Shanghai University.

\section{Construction of plant transformation vectors}

The maize U6 promoter (U6p) and U6 terminator (U6t) were amplified using gene-specific primers (see Additional file 1: Table S1 for primer sequences) and cloned into the PstI site of the pCAMBIA3301 vector with the maize codon optimized Cas9 gene (from Jinsheng Lai's lab) [12]. gRNAs designed for simplex editing and tRNA-gRNA units (TGUs) designed for multiplex editing were synthesized by Generey (Generey.com) and cloned into the Psi and $\mathrm{XbaI}$ sites between the U6 promoter and U6 terminator. The constructed plasmids, pCAMBIA3301 with
UBQp: Cas9 and U6p: gRNA or U6p: TGUs, were used for Agrobacterium-mediated maize transformation.

\section{Agrobacterium-mediated transformation of immature maize embryos}

Agrobacterium-mediated maize transformation was carried out according to Frame et al., [22]. Between 11 and 21 independent transgenic lines were generated for each transformation and genotyped with BAR specific primers (see Additional file 1: Table S1 for primer sequences).

\section{Genomic DNA extraction and PCR/Sequencing assay}

For each BAR-positive transgenic line, three individual tissue samples were used to extract genomic DNA. Maize genomic DNA was extracted with the hexadecyltrimethylammonium bromide method [23]. Target regions were amplified with specific primers pairs flanking the designed target sites (see Additional file 1: Table S1 for primer sequences) using KOD DNA polymerase (Toyobo) to detect mutagenesis at the desired sites. The PCR product was separated on a $1 \%$ agarose gel and stained using ethidium bromide. The stained gels were imaged using the Gel Doc XRS system (Bio-Rad). Selected PCR products were cloned into the pGEM-T Easy Vector (Promega) for DNA sequencing. For PCR product of each tissue sample, twenty clones were sequenced to detect stable editing.

\section{Zein extraction and quantification}

Mature kernels of either WT or MADS/Cas9 line 21 were collected from well-filled ears. Zeins were extracted from $50 \mathrm{mg}$ of dried endosperm flour according to previously described methods [24]. Extracted proteins were measured using a bicinchoninic acid protein assay kit (Pierce) according to the instructions. Measurements of all samples were replicated three times. SDS-PAGE was performed on $12 \%$ polyacrylamide gels and visualized by staining with Comassie brilliant blue (Dingguo).

\section{Results}

Strategy to engineer simplex editing and multiplex editing based on the tRNA-processing system in maize A maize codon optimized Cas9 driven by the maize ubiquitin (UBQ) promoter was inserted into pCAMBIA3301 (see Methods) to construct two binary CRISPR/Cas9 vectors for either simplex editing or multiplex editing (Fig. 1). These two vectors both contain the BAR gene as a plantselectable marker.

For the simplex editing vector, we selected and cloned a small nuclear U6 RNA promoter from maize (U6p, Chr 8: 165525624-165548023) and the corresponding U6 terminator (U6t) to facilitate the expression of the gRNA cassette in the CRISPR/Cas9 construct. The gRNA with the target sequence (gRNA spacer) is 


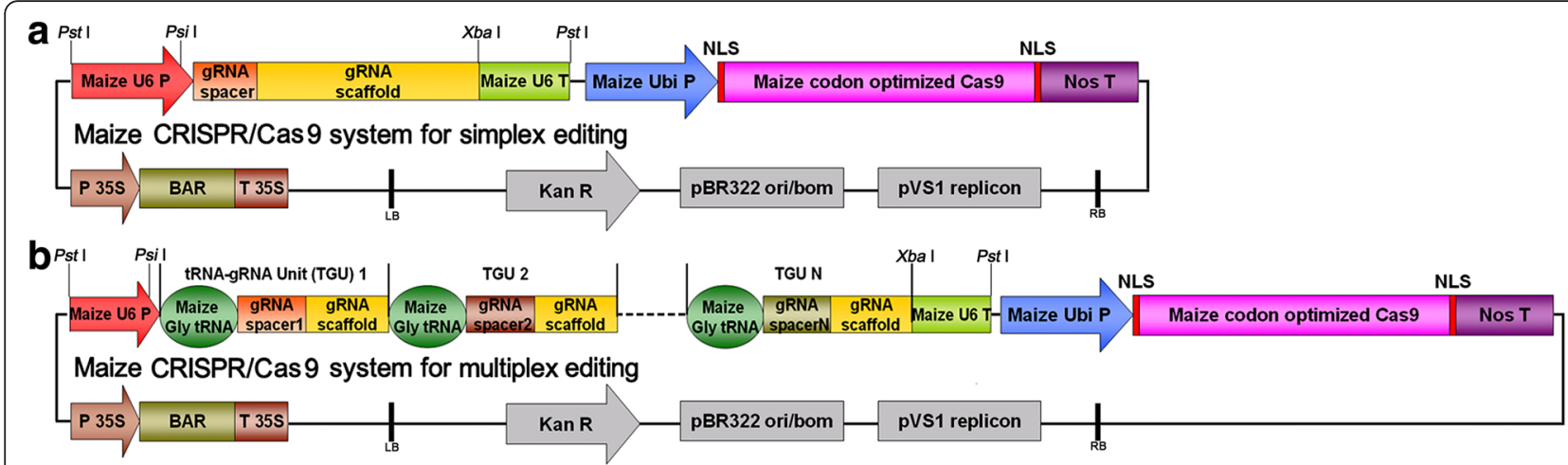

Fig. 1 Simplex and multiplex editing vector construction strategy. a \& $\mathbf{b}$ Structure of simplex and multiplex editing binary vector based on pCAMBIA3301. P, promoter; T, terminator; Ubi P, ubiquitin promoter; NLS, nuclear localization sequence; Nos T, nopaline synthase terminator; BAR, phosphinothricin $\mathrm{R}$; Kan $\mathrm{R}$, kanamycin resistance gene; LB, left border; RB, right border; TGU, tRNA-gRNA unit

transcribed from the U6 promoter with a definite transcription initiation site G nucleotide [15]. Therefore, target sequences are commonly selected for the U6 promoter by searching for 5'-GN (19) NGG motifs (NGG: protospacer adjacent motif, PAM). It was reported that the gRNA spacer with extended nucleotides at the $5^{\prime}$ end, derived from the vector ligation site, could also guide genome editing in plants [25]. It is unclear whether this kind of gRNA spacer affects the editing efficiency. Therefore, we found a Psi restriction site within the U6p to certify that the selected gRNA sequence directly followed the U6p without adding additional nucleotides at the $5^{\prime}$ end (Fig. 2a).

For the multiplex editing vector, we compacted a cluster of gRNAs with different spacers into one polycistronic gene to simultaneously produce multiple gRNAs from one primary transcript. Xie et al. [21] have proposed that the tRNA precursors, pre-tRNAs, are cleaved at specific sites in eukaryotes by RNase P and RNase Z to remove extraneous $5^{\prime}$ and $3^{\prime}$ sequences. The tRNAprocessing system is used as an intrinsic mechanism to produce different small RNAs, for example, small nucleolar RNA (snoRNA), from a single polycistronic gene. They successfully obtained multiplex editing using multiple gRNAs produced from a single synthetic gene employing the tRNA-gRNA architecture in rice. We designed multiple tRNA-gRNA units (TGUs) for the simultaneous production of numerous gRNAs to utilize the endogenous tRNA-processing system-based strategy for multiplex editing in maize (Fig. 1b). We selected the maize glycine-tRNA (Chr 5: 15452981-15473056) for the construction of the TGUs, and a spacer sequence starting with a $G$ nucleotide was inserted between the U6p and the first glycine-tRNA to certify the transcription initiation with $G$ nucleotide (Fig. 2b). The multiple TGUs (MTs) consisted of tandem repeats of tRNAgRNA and would be transcribed under the control of the U6p. The resulting gRNAs would then direct Cas9 to multiple target sites for genome editing.

\section{Effective and efficient multiplex editing in stable transgenic maize via the tRNA-processing system-based strategy}

To explore the efficiency of genome modification by our multiplex editing strategy, we synthesized three gRNAs for simplex editing and three MTs for multiplex editing.

The gRNAs for simplex editing target three transcription factors: a maize MADS gene (GRMZM2G059102), a maize MYBR gene (GRMZM2G091201), and a maize $A P 2$ gene (GRMZM2G050851). The MADS gene and the MYBR gene were both reported to be related to the maize endosperm-specific core transcription factor Opaque2 (Fig. 3a) [26, 27]. The GN (19) NGG gRNA spacer sequence selected for the targeting of $M A D S$ was at the $194 \mathrm{bp}$ of its open reading frame (ORF). The gRNA spacer sequences for $M Y B R$ and $A P 2$ were at the 237 and $33 \mathrm{bp}$ of the ORFs, respectively (Fig. 3b).

The gRNA-targeted genes for multiplex editing were a maize RPL gene (GRMZM2G024838), a maize PPR gene (GRMZM2G087226), and two reverse overlapping maize long non-coding RNAs (lncRNAs, Chr6:156117287156117915 \& Chr6:156118571-156117710) (Fig. 3a). RPL and PPR may be important for plant development in maize. The two lncRNAs were reported to be regulated by Opaque2 [26] and overlapped in opposite orientations in the maize genome. $R P L$ and $P P R$ were both targeted at two sites with a single 2 TGU construction. The two reverse overlapping lncRNAs were targeted with a single 4 TGU construction that included two sites for lncRNA1 and two sites for lncRNA2. The two GN (19) NGG sequences selected for targeting $R P L$ were at 94 and $417 \mathrm{bp}$ in its ORF, and the gRNA spacer sequences for PPR targeted 327 and $553 \mathrm{bp}$ in its ORF. The two GN (19) NGG sequences selected for targeting 


\section{a Sequence architecture for simplex editing}

5'-AAGTCGTAAAATAGTGGTGTCCAAAGAATTTCCAGGCCCAGTTGTAAAAG CTAAAATGCTATTCGAATTTCTACTAGCAGTAAGTCGTGTTTAGAAATTATT ITTTTATATACCTTTTTTCCTTCTATGTACAGTAGGACACAGTGTCAGCGCC GCGTTGACGGAGAATATTTGCAAAAAAGTAAAAGAGAAAGTCATAGCGG Maize U6 promoter (chr8:165525624-165546023) CGTATGTGCCAAAAACTTCGTCACAGAGAGGGCCATAAGAAACATGGCC CACGGCCCAATACGAAGCACCGCGACGAAGCCCAAACAGCAGTCCGTAG GTGGAGCAAAGCGCTGGGTAATACGCAAACGTTTTGTCCCACCTTGACTA ATCACAAGAGTGGAGCGTACCTTATAAACCGAGCCGCAAGCACCGAATT

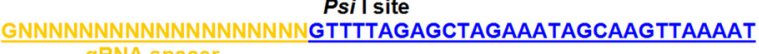
AAGGCTAGTCCGTTATCAACTTGAAAAAGTGGCACCGAGTCGGTGCTTTT gRNA scaffold

ITTTCTAGAGCGGATTCGCACATATGCCCTCGGAGATCGCATTTTGATCCT Xbal site

TCTGCGCCAGTTTTCATTAACAATTCAGTCCTCGCCAATTCTTGCCGGCTC

TGTACCAGAACCTTTTGTTCGCTGTTTGCCGATTGGCCCTTGCCCTTGCAA ACATGGGATTTCGCGCACTGTCCCTCTTATTATCATTTTCCTCGCATATGC CCTCGAAGATCTCACTTTGGTTCTTCTGCTCTATTTTTTTTCTTAACGATTTA GCCCTCGC -3'

b Sequence architecture for multiplex editing

5'-AAGTCGTAAAATAGTGGTGTCCAAAGAATTTCCAGGCCCAGTTGTAAAAGCT AAAATGCTATTCGAATTTCTACTAGCAGTAAGTCGTGTTTAGAAATTATTTTTT IATATACCTTTTTTCCTTCTATGTACAGTAGGACACAGTGTCAGCGCCGCGTT GACGGAGAATATTTGCAAAAAAGTAAAAGAGAAAGTCATAGCGGCGTATGT GCCAAAAACTTCGTCACAGAGAGGGCCATAAGAAACATGGCCCACGGCCC AATACGAAGCACCGCGACGAAGCCCAAACAGCAGTCCGTAGGTGGAGCAA AGCGCTGGGTAATACGCAAACGTTTTGTCCCACCTTGACTAATCACAAGAG TGGAGCGTACCTTATAAACCGAGCCGCAAGCACCGAATT TTAGTAACA TTC Psi l site

AGAGCACCAGTGGTCTAGTGGTAGAATAGTACCCTGCCACGGTACAGACCC Maize Gly-tRNA (chr5:15452981-15473056) sequence GGGTTCGATTCCCGGCTGGTGCA GNNNNNNNNNNN NNNNNNNN GTTTTAG AGCTAGAAATAGCAAGTTAAAATAAGGCTAGTCCGTTATCAACTTGAAAAAG gRNA scaffold
TGGCACCGAGTCGGTGCTTCAGAGTAGTGGTAGAATAGTA $\frac{\text { TGGCACCGAGTCGGTGCTTCAGAGCACCAGTGGTCTAGTGGTAGAATAGTA }}{\text { Maize Gly-tRNA sequence }}$ CCCTGCCACGGTACAGACCCGGGTTCGATTCCCGGCTGGTGCAGNNNNNN NNNNNNNNNNNNNGTTTTAGAGCTAGAAATAGCAAGTTAAAATAAGGCTAG gRNA spacer for Gene2 2 gRNA scaffold TCCGTTATCAACTTGAAAAAGTGGCACCGAGTCGGTGC..................TTCA GAGCACCAGTGGTCTAGTGGTAGAATAGTACCCTGCCACGGTACAGACCCG Maize Gly-tRNA sequence GGTTCGATTCCCGGCTGGTGCAGNNNNNNNNNNNNNNNNNNNGTTTTAGA gRNA spacer for GeneN

GCTAGAAATAGCAAGTTAAAATAAGGCTAGTCCGTTATCAACTTGAAAAAGT

gRNA scaffold
GGCACCGAGTCGGTGCTTTTTTTTCTAGAGCGGATTCGCACATATGCCCTC Xbal site GGAGATCGCATTTTGATCCTTCTGCGCCAGTTTTCATTAACAATTCAGTCCT CGCCAATTCTTGCCGGCTCTGTACCAGAACCTTTTGTTCGCTGTTTGCCGAT TGGCCCTTGCCCTTGCAAACATGGGATTTCGCGCACTGTCCCTCTTATTATC ATTTTCCTCGCATATGCCCTCGAAGATCTCACTTTGGTTCTTCTGCTCTATTT

ITTTTCTTAACGATTTAGCCCTCGC -3'

Fig. 2 gRNA for simplex editing and the tRNA-gRNA units for multiplex editing sequence design strategy. $\mathbf{a}$ \& $\mathbf{b}$ The specific sequence of the core elements of the simplex editing and multiplex editing vectors designed for the usage in maize, which can be directly used for broad gene knock-out in further maize genetics and maize breeding studies 


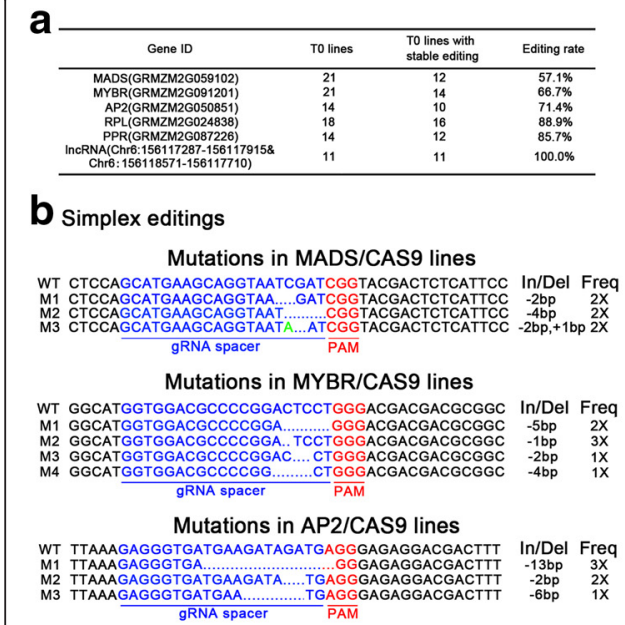

C Multiplex editings

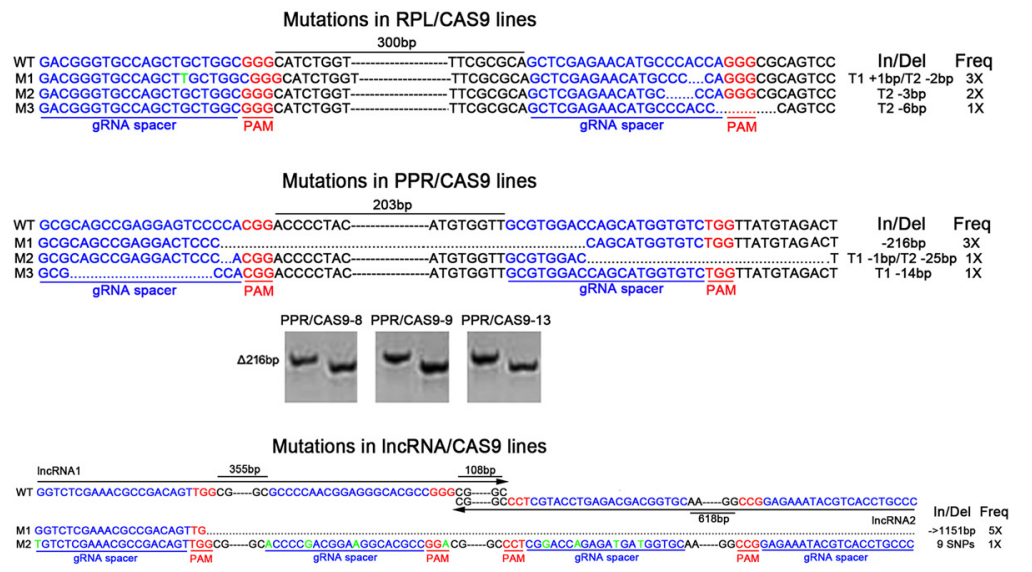

Fig. 3 Simultaneous sequence editing results of simplex and multiplex editing. a Targeted mutation efficiency of simplex and multiplex edited plants. Editing rate showed the percentage of TO transgenic lines with stable heritable editing within all TO lines. $\mathbf{b}$ The DNA sequence of the simplex edited genes is provided. The 20-bp gRNA spacer sequence for the Cas9/gRNA complex is in blue, and the PAM site is in red. Deleted nucleotides are depicted as dots, and inserted nucleotides are shown in green. The lengths of the insertions and/or deletions (In/Del) and the frequencies (Freq) are presented. WT, wild type; M1, mutant1. c The DNA sequence of the multiplex edited genes is provided. The 20-bp gRNA spacer sequence for the Cas9/gRNA complex is in blue, and the PAM site is in red. Deleted nucleotides are depicted as dots, and inserted nucleotides are shown in green. The lengths of the insertions and/or deletions (In/Del) and the frequencies (Freq.) are presented. WT, wild type; M1, mutant1; T1, target1. Gel pictures show PCR products amplified from PPR/CAS9-8, 9 and -13 lines. Left lane, WT; right lane, mutant

lncRNA1 were at 62 and $440 \mathrm{bp}$, and gRNA spacer sequences targeting lncRNA2 were at the 52 and $693 \mathrm{bp}$ (Fig. 3c).

We used conventional Agrobacterium-mediated transformation to produce the stable transgenic lines for the six constructs and evaluated the efficacy of our simplex and multiplex editing system. Twenty-one independent transgenic lines were generated for $M A D S$ and $M Y B R$. Fourteen independent transgenic lines were generated for $A P 2$ and $P P R, 18$ independent transgenic lines were generated for $R P L$, and 11 independent transgenic lines were generated for the lncRNAs (Fig. 3a).

Mutagenesis frequency was examined in the T0 generation. Inheritance of the edited sites in T0 transgenic lines was desired for maize genetics and breeding researches and stable editing throughout the whole transgenic plant is heritable. For each BAR-positive transgenic line, three individual tissue samples were used to extract genomic DNA. Target regions were amplified with specific primers pairs flanking the designed target sites. The PCR product was cloned into the pGEM-T Easy Vector. For the three tissue samples of each transgenic line, twenty clones for PCR product of each tissue sample were sequenced to detect stable editing.

In the T0 generation of the MADS/CAS9 plants, $57.1 \%$ (12 lines) carried stable editing including In/ Dels and SNPs. The MADS/CAS9-21 transgenic plant had a biallelic mutation. We found stable mutations in $66.7 \%$ (14 lines) of the T0 MYBR/CAS9 plants, and $71.4 \%$ (10 lines) of the AP2/CAS9 T0 lines were mutants (Fig. $3 \mathrm{a}$ and $\mathrm{b}$ ). Higher mutagenesis efficiency was achieved in the T0 generation of the multiplex editing lines than in the simplex editing lines. In the RPL/ CAS9 plants, $88.9 \%$ (16 lines) of T0 lines had stable mutations. However, the chromosomal-fragment deletion between two targets that can be achieved by the tRNA-processing system as reported by Xie et al. [21] was not detected in the RPL/CAS9 plants. This indicates that the MTs do not always operate on both targets simultaneously. The PPR/CAS9 plants had stable mutations in $85.7 \%$ (12 lines) of T0 lines. The chromosomal-fragment deletion between target1 and target 2 was detected in the PPR/CAS9-8, 9 and 13 T0 lines (Fig. 3c). Interestingly, these three lines also carry biallelic mutations. For the lncRNAs targeted from a single 4 TGU construct that included two sites for lncRNA1 and two sites for lncRNA2, $100 \%$ (11 lines) of T0 lines had mutations. Surprisingly, a large region chromosomal-fragment deletion (about $2 \mathrm{~kb}$ ) beyond the sequence between targets was detected in several transgenic lines (Fig. 3c), and there were only SNPs at target sites in other transgenic lines. Our data also demonstrated that the tRNA-processing system for multiplex editing not only increased the targeted sites but also significantly enhanced mutagenesis efficiency in maize $(p$-value $=0.021)$. 


\section{Generation of phenotypic mutants}

The biallelic transgenic line MADS/CAS9-21 for MADS gene and transgenic lines PPR/CAS9-9 and -13 for PPR gene were selected for further phenotypic analysis.

Zeins are the most abundant storage proteins in maize kernels, and are encoded by different classes of genes. MADS (GRMZM2G059102) was reported to interact with Opaque2 and activate zein gene promoters. In MADS $R N A i$ kernels, the expression of the $22-\mathrm{kD} \alpha$-zein genes, the $19-\mathrm{kD} \alpha$-zein genes and the $50-\mathrm{kD} \gamma$-zein gene decreased. Relative differences in these zein protein contents can be observed in MADS RNAi kernels [27]. Quantitative analysis showed that zeins were significantly decreased (12.5 \%) in MADS/CAS9-21 kernels (Fig. 4a). We also observed differences in relative contents of the $22-\mathrm{kD} \alpha-$ zein, the $19-\mathrm{kD} \alpha$-zein and the $50-\mathrm{kD} \gamma$-zein proteins between the wild type and MADS/CAS9-21 kernels through SDS-PAGE, while the contents of $27 \mathrm{kD} \gamma$-zein and $14 \mathrm{kD}$ $\beta$-zein proteins were not affected (Fig. 4b).

In plants, most respiratory chain related proteins are expressed by mitochondrial genome and undergo posttranscriptional processes regulated by nuclear genome expressed factors, including pentatricopeptide repeat (PPR) proteins. PPRs were reported to affect the endosperm, embryo and seedling development [28, 29]. We observed a significant developmental delay of the PPR/ CAS9-9 and -13 plants compared to wild type at 35 days after pollination (35 DAP, Fig. 4c). a

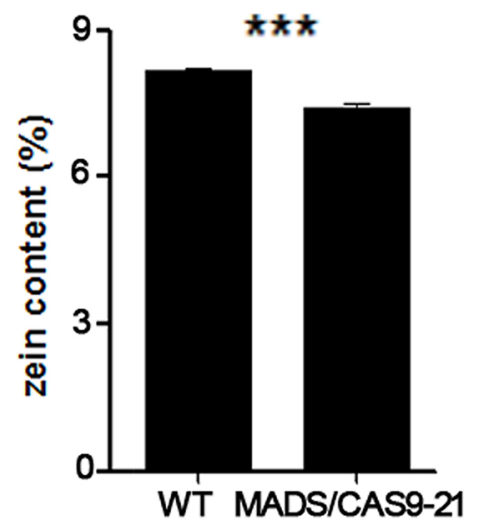

b

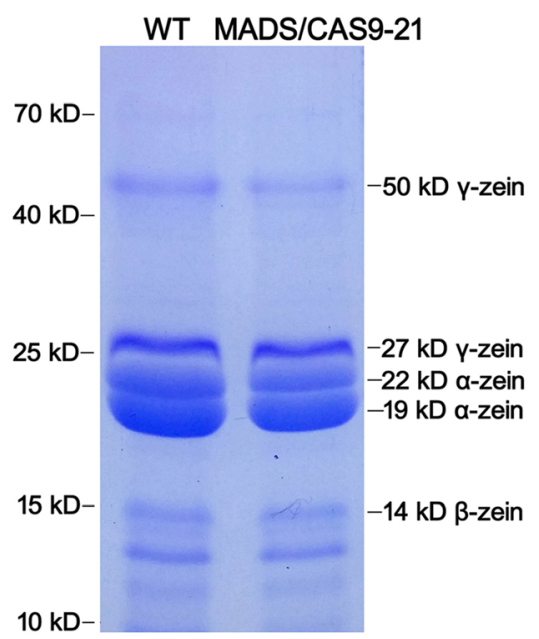

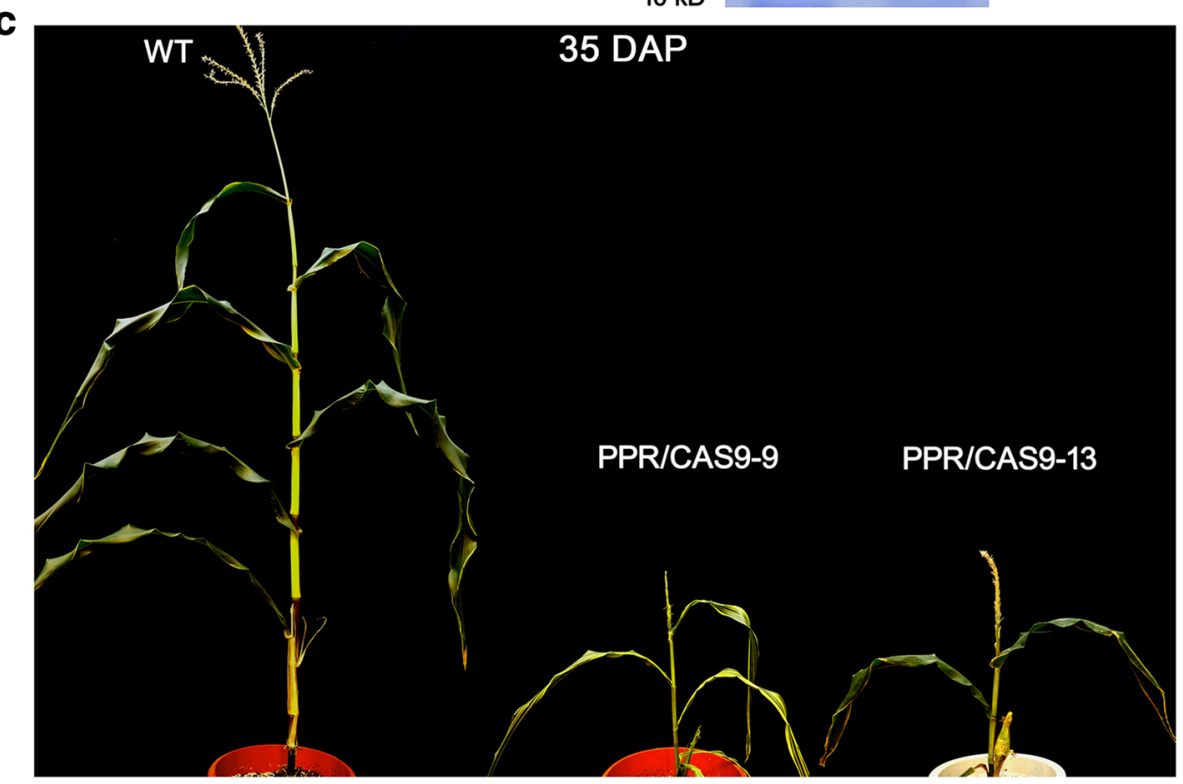

Fig. 4 Phenotypic variations of simplex and multiplex editing mutants generated from the CRISPR/Cas9 targeting. a Comparison of zein protein contents from WT and MADS/CAS9 line 21 mature endosperm. The measurements were done on per $50 \mathrm{mg}$ of dried endosperm. Error bars

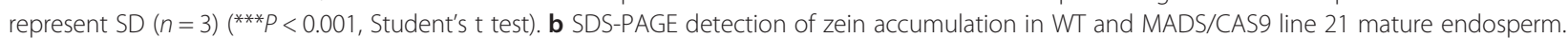
c Mature (35 DAP) WT and T0 transgenic maize plants of the mutant lines PPR/CAS9-9 and -13 


\section{Discussion}

Maize is one of the most important cereal crops in the world. High efficient and accurate gene modification would benefit maize genetics study and breeding. We provided a framework to design, synthesize and use multiple tRNA-gRNA units for multiplex gene editing with CRISPR/Cas9 in maize (Figs. 1 and 2). These multiple tRNA-gRNA units were expressed under the control of the selected maize Pol III promoter (maize U6p). In this study, we successfully produced simultaneous mutagenesis of multiple genomic loci or deletion of short chromosomal fragments (Fig. 3). Our results showed that the optimized tRNA-processing system-based strategy is a robust and efficient tool for multiply targeted genome modification in maize. Our results also demonstrated that targeting one gene with two gRNAs using multiple tRNA-gRNA units greatly increased the efficiency of gene knock-out in maize. Compared to the parallel simplex editing system, the tRNA-processing strategy enables significantly higher editing efficiency ( $p$ value $=0.021$, Fig. 3). Given the extremely large number of tRNA genes and the fact that RNase P and RNase $\mathrm{Z}$ precisely recognize RNA substrates with tRNA-like structures [21, 30], there are many other choices of tRNA sequences to be embedded in the multiple tRNAgRNA units in maize, implying higher efficiency of gene knock-out might be achieved with advanced design.

The mutation ratio of different construction for targeting different genes was varied, ranging from 57.1 to $71.4 \%$ for simplex editing and $85.7-100 \%$ for multiplex editing. There might be some factors regulating the mutation ratio, including the efficiency of gRNA to search and target to the specific site and different T-DNA insertion site in the genome [31]. Moreover, the mutation efficiency of CRIPSR/Cas9 system is variable in different plant species [32, 33]. The tRNA-processing systembased strategy enables the generation of many doublestrand breaks (DSB) in genomic DNA. It may provide an efficient tool to help dissect the molecular process of chromosomal deletion. Due to the differences in the delivery, expression and activity of gRNAs and Cas9, it is not surprising to see some discrepancies in fragmentdeletion frequency between stable transgenic plants containing different multiple tRNA-gRNA units (Fig. 3). Compared with RPL, the gRNA spacers selected for PPR were physically closer, approximately $200 \mathrm{bp}$ apart in PPR and 300 bp apart in RPL, and had a higher sequence similarity, approximately $25 \%$ similarity in PPR and $45 \%$ similarity in RPL. The accurate chromosomal fragment deletion between two targets only existed in PPR/CAS9 lines but not in RPL/CAS9 lines (Fig. 3). Based on our results, we propose an improved sequence selection of gRNA spacers for high efficient chromosomal fragment deletion in which the distance between the two gRNA spacers should not be too long and the sequences of the two gRNA spacers should have high identity. gRNA targets of this type might have similar chromosome structure, binding ability, delivery and activity, causing generation of DSBs in the genome at the same time. The two reverse overlapping lncRNAs were targeted with a single 4 TGU construction that including two sites for lncRNA1 and two sites for lncRNA2. In plants transformed with this construct, a long-distance deletion beyond the sequence between the targets was observed. The way too high density and number of targets may have been the cause of this unintended mutation. As only SNPs at target sites were not useful for the knock-out of lncRNAs, long chromosomal fragment deletion fulfilled the complete abolishment of lncRNA function. Further more, it is also indicated that up to 4 TGUs design of tRNA-processing system-based strategy can still deliver gene modification in maize. Higher multiple TGUs design might also work in maize.

\section{Conclusions}

This is the first report of successful multiplex gene editing using the tRNA-processing system in maize. This optimized tRNA-processing system-based strategy for maize can be broadly used for stable complete gene knock-out in the future. We propose that the tRNAprocessing system-based strategy improves the efficiency of the CRISPR/Cas9 editing in maize. Additionally, advanced sequence selection of gRNA spacers to generate DSBs in the genome at the same time increases the efficiency and accuracy of chromosomal fragment deletions for complete abolishment of gene function especially lncRNAs, which is important for the enhancement of maize genetic research and breeding.

\section{Additional file}

\section{Additional file 1: Table S1. The primers used in the experiments.} (PDF $7 \mathrm{~kb}$ )

\section{Abbreviations \\ CRISPR/Cas9, clustered regularly interspersed short palindromic repeat/ CRISPR-associated protein 9; gRNA, guide RNA; MT, multiple TGU; ORF, open reading frame; PAM, protospacer adjacent motif; TGU, tRNA-gRNA unit; U6p, U6 promoter; U6t, U6 terminator}

\section{Acknowledgements}

This work was supported by the Ministry of Science and Technology of China (2014CB138204) and the National Natural Sciences Foundation of China (31271733). We thank Dr. Jinsheng Lai (China Agriculture University) for the application of maize codon optimized Cas9 gene.

\section{Availability of data and materials}

The datasets supporting the conclusions of this article are included within the article and its additional file. 


\section{Authors' contributions}

$\mathrm{RS}$, WQ designed the experiment. WQ, TZ, ZT, CL and WZ performed the experiments. WQ analyzed the data. WQ and RS wrote the article. All authors read and approved the final manuscript.

\section{Competing interests}

The authors declare that they have no competing interests.

\section{Consent for publication}

Not applicable.

\section{Ethics approval and consent to participate}

Not applicable.

\section{Author details}

'Shanghai Key Laboratory of Bio-Energy Crops, School of Life Sciences, Shanghai University, 333 Nanchen Road, Shanghai 200444, China. ${ }^{2}$ National Maize Improvement Center of China, China Agricultural University, Beijing 100193, China. ${ }^{3}$ Coordinated Crop Biology Research Center (CBRC), Beijing 100193, China

\section{Received: 13 June 2016 Accepted: 2 August 2016}

\section{Published online: 11 August 2016}

\section{References}

1. Wiedenheft B, Sternberg SH, Doudna JA. RNA guided genetic silencing systems in bacteria and archaea. Nature. 2012;7385:331-8.

2. Jinek M, Chylinski K, Fonfara I, Hauer M, Doudna JA, Charpentier E. A programmable dual-RNA-guided DNA endonuclease in adaptive bacterial immunity. Science. 2012;6096:816-21.

3. Mali P, Yang L, Esvelt KM, Aach J, Guell M, DiCarlo JE, Norville JE, Church GM. RNA-guided human genome engineering via Cas9. Science. 2013;6121:823-6.

4. DiCarlo JE, Norville JE, Mali P, Rios X, Aach J, Church GM. Genome engineering in Saccharomyces cerevisiae using CRISPR-Cas systems. Nucleic Acids Res. 2013;7:4336-43.

5. Hwang WY, Fu Y, Reyon D, Maeder ML, Tsai SQ, Sander JD, Peterson RT, Yeh $J R$, Joung JK. Efficient genome editing in zebrafish using a CRISPR-Cas system. Nat Biotechnol. 2013;3:227-9.

6. Gratz SJ, Cummings AM, Nguyen JN, Hamm DC, Donohue LK, Harrison MM, Wildonger J, O'Connor-Giles KM. Genome engineering of Drosophila with the CRISPR RNA-guided Cas9 nuclease. Genetics. 2013;194:1029-35.

7. Cho SW, Kim S, Kim JM, Kim JS. Targeted genome engineering in human cells with the Cas9 RNA-guided endonuclease. Nat Biotechnol. 2013;3:230-2.

8. Jiang W, Zhou H, Bi H, Fromm M, Yang B, Weeks DP. Demonstration of CRISPR/Cas9/sgRNA-mediated targeted gene modification in Arabidopsis, tobacco, sorghum and rice. Nucleic Acids Res. 2013:41:e188.

9. Kumar V, Jain M. The CRISPR-Cas system for plant genome editing: advances and opportunities. J Exp Bot. 2015;66:47-57.

10. Liang Z, Zhang K, Chen K, Gao C. Targeted mutagenesis in Zea mays using TALENs and the CRISPR/Cas system. J Genet Genomics. 2014:41:63-8.

11. Svitashev S, Young JK, Schwartz C, Gao H, Falco SC. Cigan AMTargeted Mutagenesis, Precise Gene Editing, and Site-Specific Gene Insertion in Maize Using Cas9 and Guide RNA. Plant Physiol. 2015;169:931-45.

12. Zhu J, Song N, Sun S, Yang W, Zhao H, Song W, Lai J. Efficiency and Inheritance of targeted mutagenesis in maize using CRISPR-Cas9. J Genet Genomics. 2016;43:25-36.

13. Feng C, Yuan J, Wang R, Liu Y, Birchler JA, Han F. Efficient targeted genome modification in maize using CRISPR/Cas9 System. J Genet Genomics. 2016; 43:37-43.

14. Jacobs TB, LaFayette PR, Schmitz RJ, Parrott WA. Targeted genome modifications in soybean with CRISPR/Cas9. BMC Biotechnol. 2015;15:16.

15. Cong L, Ran FA, Cox D, Lin S, Barretto R, Habib N, Hsu PD, Wu X, Jiang W, Marraffini LA, et al. Multiplex genome engineering using CRISPR/Cas systems. Science. 2013;6121:819-23.

16. Wang $H$, Yang H, Shivalila CS, Dawlaty MM, Cheng AW, Zhang F, Jaenisch R. One-step generation of mice carrying mutations in multiple genes by CRISPR/Cas-mediated genome engineering. Cell. 2013;4:910-8.

17. Li JF, Norville JE, Aach J, McCormack M, Zhang D, Bush J, Church GM, Sheen J. Multiplex and homologous recombination-mediated genome editing in Arabidopsis and Nicotiana benthamiana using guide RNA and Cas9. Nat Biotechnol. 2013;31(8):688-91.
18. Shan Q, Wang Y, Li J, Zhang Y, Chen K, Liang Z, Zhang K, Liu J, Xi JJ, Qiu JL, Gao C. Targeted genome modification of crop plants using a CRISPR-Cas system. Nat Biotechnol. 2013;31(8):686-8.

19. Xing HL, Dong L, Wang ZP, Zhang HY, Han CY, Liu B, Wang XC, Chen QJ. A CRISPR/Cas9 toolkit for multiplex genome editing in plants. BMC Plant Biol. 2014;14:327.

20. Ma X, Zhang Q, Zhu Q, Liu W, Chen Y, Qiu R, Wang B, Yang Z, Li H, Lin Y, Xie Y, Shen R, Chen S, Wang Z, Chen Y, Guo J, Chen L, Zhao X, Dong Z, Liu YG. A Robust CRISPR/Cas9 System for Convenient, High-Efficiency Multiplex Genome Editing in Monocot and Dicot Plant. Mol Plant. 2015;8:1274.

21. Xie K, Minkenberg B, Yang Y. Boosting CRISPR/Cas9 multiplex editing capability with the endogenous tRNA-processing system. Proc Natl Acad Sci U S A. 2015;112:3570-5.

22. Frame BR, Shou H, Chikwamba RK, Zhang Z, Xiang C, Fonger TM, Pegg SEK, Li B, Nettleton DS, Pei D. Agrobacterium tumefaciens-mediated transformation of maize embryos using a standard binary vector system. Plant Physiol. 2002;129:13-22.

23. Gao H, Smith J, Yang M, Jones S, Djukanovic V, Nicholson MG, West A, Bidney D, Falco SC, Jantz D, et al. Heritable targeted mutagenesis in maize using a designed endonuclease. Plant J. 2010;61:176-87.

24. Wallace JC, Lopes MA, Paiva E, Larkins BA. New methods for extraction and quantitation of zeins reveal a high content of $y$-zein in modified opaque-2 maize. Plant Physiol. 1990;92:191-6.

25. Xie K, Yang Y. RNA-guided genome editing in plants using a CRISPR-Cas system. Mol Plant. 2013;6(6):1975-83.

26. Li C, Qiao Z, Qi W, Wang Q, Yuan Y, et al. Genome-Wide Characterization of cis-Acting DNA Targets Reveals the Transcriptional Regulatory Framework of Opaque2 in Maize. Plant Cell. 2015;27:532-45.

27. Qiao Z, Qi W, Wang Q, Feng Y, Yang Q, Zhang N, Wang S, Tang Y, Song R. ZmMADS47 regulates zein gene transcription through interaction with Opaque2. Plos Genet. 2016. doi:10.1371/journal.pgen.1005991.

28. Li XJ, Zhang YF, Mm H, Sun F, Shen Y, Xiu ZH, Wang XM, Chen ZL, Sun S, Small I, Tan BC. Small kernel 1 encodes a pentatricopeptide repeat protein required for mitochondrial nad7 transcript editing and seed development in maize (Zea mays) and rice (Oryza sativa). Plant J. 2014;79:797-809.

29. Sun F, Wang XM, Bonnard G, Shen Y, Xiu Z, Li X, Gao D, Zhang Z, Tan BC. Empty pericarp7 encodes a mitochondrial E-subgroup pentatricopeptide repeat protein that is required for $\mathrm{CCMF}_{N}$ editing, mitochondrial function and seed development in maize. Plant J. 2015;84:283-95.

30. Forster AC, Altman S. External guide sequences for an RNA enzyme. Science. 1990;249(4970):783-6.

31. Johnson RA, Gurevich V, Filler S, Samach A, Levy AA. Comparative assessments of CRISPR-Cas nucleases' cleavage efficiency in planta. Plant Mol Biol. 2015:87:143e156.

32. Fauser F, Schiml S, Puchta H. Both CRISPR/Cas-based nucleases and nickases can be used efficiently for genome engineering in Arabidopsis thaliana. Plant J. 2014;79:348e359

33. Zhang $H$, Zhang J, Wei $P$, Zhang B, Gou F, Feng Z, Mao Y, Yang L, Zhang $H$ $\mathrm{Xu} N$, Zhu J. The CRISPR/Cas9 system produces specific and homozygous targete gene editing in rice in one gerneration. Plant Biotech J. 2014;12: $797 \mathrm{e} 807$.

\section{Submit your next manuscript to BioMed Central} and we will help you at every step:

- We accept pre-submission inquiries

- Our selector tool helps you to find the most relevant journal

- We provide round the clock customer support

- Convenient online submission

- Thorough peer review

- Inclusion in PubMed and all major indexing services

- Maximum visibility for your research

Submit your manuscript at www.biomedcentral.com/submit
Biomed Central 